\title{
Patterned Geometries and Hydrodynamics at the Vortex Bose Glass Transition
}

\author{
M. Cristina Marchetti ${ }^{\dagger}$ and David R. Nelson* \\ * Lyman Laboratory of Physics, Harvard University, Cambridge, MA 01238 \\ ${ }^{\dagger}$ Physics Department, Syracuse University, Syracuse, NY 13244
}

(August 12, 2018)

\begin{abstract}
Patterned irradiation of cuprate superconductors with columnar defects allows a new generation of experiments which can probe the properties of vortex liquids by confining them to controlled geometries. Here we show that an analysis of such experiments that combines an inhomogeneous Bose glass scaling theory with the hydrodynamic description of viscous flow of vortex liquids can be used to infer the critical behavior near the Bose glass transition. The shear viscosity is predicted to diverge as $\left|T-T_{B G}\right|^{-z}$ at the Bose glass transition, with $z \simeq 6$ the dynamical critical exponent.
\end{abstract}

In the mixed state of cuprate superconductors the magnetic field is concentrated in an array of flexible flux bundles that, much like ordinary matter, can form crystalline, liquid and glassy phasest. The dynamics of the flux-line array determines the resistive properties of the material and has therefore been the focus of much attention. Novel types of glasses are also possible because of pinning in disordered samples2. In particular, the introduction of columnar damage tracks by heavy-ion irradiation yields a low-temperature "Bose glass" phase in which every vortex is trapped on a columnar defect 3 and the pimning efficiency of vortex lines is strongly enhanced 4 6. At high temperatures the vortices delocalize in an entangled flux-line liquid. The high temperature liquid transforms into a Bose glass via a second order phase transition at $T_{B G}$, characterized by universal critical exponents 3 . We show here that there are very strong divergences in the vortex shear viscosity and other transport coefficients as this transition is approached from the liquid, similar to behavior conjectured for glass transitions in ordinary forms of matter, and propose experiments which test our predictions. Vortex matter with columnar defects thus provides a concrete example of a glassy phase accessed via a genuine second order phase transition and characterized by universal critical exponents.

The Bose glass transition has been studied theoretically by viewing the vortex line trajectories as the world lines of two-dimensional quantum mechanical particless 3 . The thickness of the superconducting sample corresponds to the inverse temperature of the ficticious quantum particles. In thick samples the physics of vortex lines pinned by columnar defects becomes equivalent to the low temperature properties of two-dimensional bosons with point disorder. The low temperature phase is a Bose glass where the vortices behave like localized bosons. It has vanishing linear resistivity and an infinite tilt modulus 3 . The entangled flux liquif phase is resistive and corresponds to a boson superfluid 9 .

Although an exact theory of the continuous transition at $T_{B G}(B)$ from the Bose glass to the entangled flux liquid (or "superfluid") is not available, most physical prop- erties can be described via a scaling theory terms of just two undetermined critical exponents 10.11 . In the low temperature Bose glass each flux line is localized in the vicinity of one or more columnar pins. Its excursion in the direction perpendicular to the applied field is characterized by a correlation length that diverges at $T_{B G}, l_{\perp}(T) \sim\left|T-T_{B G}\right|^{-\nu_{\perp}}$. There is also a diverging correlation length along the applied field direction (here the $z$ direction), $l_{\|}(T) \sim\left|T-T_{B G}\right|^{-\nu_{\|}}$, where $\nu_{\|}=2 \nu_{\perp} 11$. The time scale $\tau$ for relaxation of a fluctuation of size $l_{\perp}$ is assumed to diverge with a critical exponent $z, \tau \sim l_{\perp}^{z} \sim\left|T-T_{B G}\right|^{-z \nu_{\perp} \text { 3. The universal }}$ critical exponents as determined by the most recent simulations are $\nu_{\perp} \simeq 1$ and $z \simeq 4.6 \pm 212$. Scaling can then be used to relate physical quantities to these diverging length and time scales. In particular, the resistivity $\rho(T)$ for currents applied in the $a b$ plane is predicted to vanish

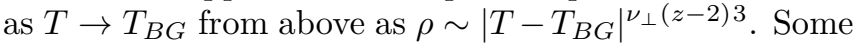
predictions of the scaling theory have been tested experimentally, but there are as yet no direct measurements of the transport coefficients usually associated with glass transitions in conventional forms of matter, such as the shear viscosity. As we shall see, the behavior of the shear viscosity is determined by the dynamical critical exponent $z$ that controls the divergence of the relaxation time in the Bose glass phase. A measurement of the shear viscosity would provide a direct probe of the diverging relaxation time associated with glassy behavior 13 .

Patterned irradiation of cuprate superconductors with columnar defects allows for a new generation of experiments that may in fact provide a direct probe of yiscous critical behavior near the Bose glass transition 14. By starting with a clean sample, at temperatures such that point disorder is negligible, it is possible to selectively irradiate regions of controlled geometry. An example is shown in Fig. 1. The side regions have been heavily irradiated, and are characterized by a high matching field $B_{\phi}^{(2)}$ and transition curve $T_{B G}^{(2)}$, while the channel is lightly irradiated with a lower matching field $B_{\phi}^{(1)}<B_{\phi}^{(2)}$ and transition curve $T_{B G}^{(1)}$. When $T_{B G}^{(1)}<T_{B G}<T_{B G}^{(2)}$, the flux array in the channel is in the liquid state, while the 
contacts are in the Bose glass phase. Flow in the resistive flux liquid region is impeded by the "Bose-glass contacts" at the boundaries, as the many trapped vortices in these regions provide an essentially impenetrable barrier for the flowing vortices. As discussed in Ref. 15, the pinning at the boundaries propagates into the liquid channels by a viscous length $\delta$ that depends on the flux liquid viscosity. As the temperature is lowered at constant field, so that the Bose glass transition $T_{B G}^{(1)}$ of the liquid region is approached from above (Fig. 2) the growing Bose glass correlations increase $\delta$ and strongly suppress the flow in the channel and the associated flux flow voltage drop across the channel.



FIG. 1. A weakly irradiated channel (white region) where the flux liquid is sandwiched between two heavily irradiated Bose-glass contacts (shaded regions). A current $J$ applied across the channel yields flux motion along the channel. The reduced field profiles given by Eq. (9) are shown for a few values of $\delta / L$ and can be measured by a series of voltage taps.

In this paper we analyze experiments with flux flow in such confined geometries by combining the predictions of the Bose glass scaling theory - generalized to the spatially inhomogeneous case - with the hydrodynamics of viscous flow of vortex liquids 15 . Our analysis shows that the viscous length $\delta$ controlling boundary pinning is just the Bose-glass localization length, $l_{\perp}$, and therefore provides a prescription for measuring the Bose glass scaling near the transition. Both flow in the channel geometry sketched in Fig. 1 and in the Corbino disk geometry (Fig. 3) used recently by López et al 16 is discussed. Such experiments can be used to extract the critical behavior of various transport coefficients and map out the entire critical region. In particular, the flux liquid shear viscosity is predicted to diverge as $\left|T-T_{B G}\right|^{-z}$ at the Bose glass transition. Because $z \simeq 4.6 \pm 2.0$, this powerful divergence is reminiscent of the Vogel-Fulcher behavior $\eta \sim \exp \left[c /\left(T-T_{g}\right)\right]$ conjectured for glass transitions in conventional forms of matter.

The Bose glass scaling theory summarized earlier is easily generalized to the case of spatially inhomogeneous flow in constrained geometries. Considering for simplicity the channel geometry, a generalized scaling ansatz for the local electric field from flux motion at position $x$ in a channel of thickness $L$ takes the form

$$
E(T, J, x, L)=b^{-(1+z)} E\left(b^{1 / \nu_{\perp}} t, \frac{b^{\nu_{\perp}} b^{\nu_{\|} J \phi_{0}}}{c k_{B} T}, \frac{x}{b}, \frac{L}{b}\right),
$$

where $b>1$ is the length scaling parameter and $t=$ $\left|T-T_{B G}\right| / T_{B G}$ the reduced temperature. This ansatz follows from the usual assumption that the continuous transition is described by a single diverging length scale and the homogeneity condition on the relevant physical quantities at the transition (see, e.g., Refs. 3.11).

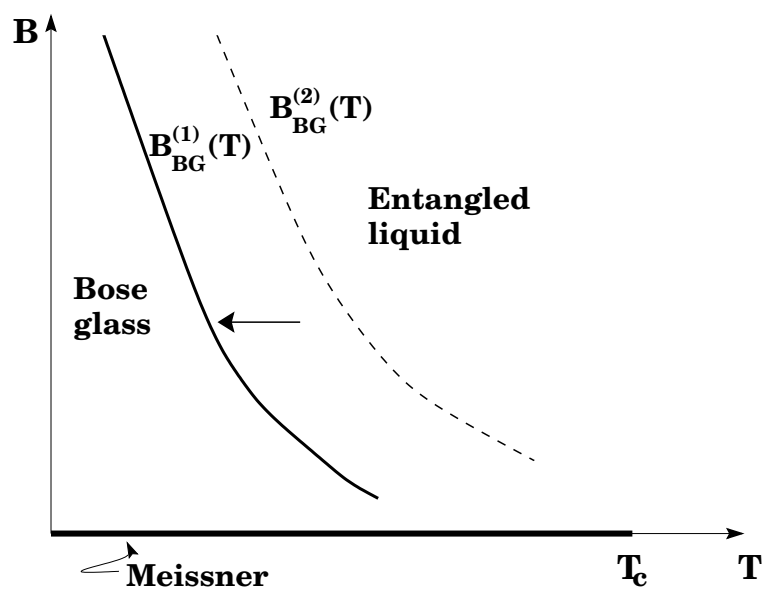

FIG. 2. A sketch of the $(B, T)$ phase diagram for the flux array in the weakly irradiated channel region. The heavy line $B_{B G}^{(1)}(T)$ denotes the continuous transition from the Bose glass to the entangled liquid. Also shown is the location $B_{B G}^{(2)}(T)$ of the Bose glass transition line in the heavily irradiated contacts. When a field $B_{B G}^{(1)}(T)<B<B_{B G}^{(2)}(T)$ is applied, the flux array in the channel is in the liquid state, while the contacts are in the Bose glass phase. By decreasing the temperature at constant field as indicated by the arrow, the Bose glass transition of the channel region is approached from above.

The response in the Bose glass is generally nonlinear in the applied current $J$. By choosing $b=t^{-\nu_{\perp}} \sim l_{\perp}(T)$ we obtain

$$
E(T, J, x, L)=l_{\perp}^{-(1+z)} E\left(1, \frac{l_{\perp} l_{\|} J \phi_{0}}{c k_{B} T}, \frac{x}{l_{\perp}}, \frac{L}{l_{\perp}}\right) .
$$

In the entangled flux liquid the response is linear at small current. Upon expanding the right hand side of Eq. (2) we obtain for $J \rightarrow 0$

$$
E(J \rightarrow 0, x, L) \simeq \rho_{0}\left(\frac{l_{\perp}}{a_{0}}\right)^{2-z} J \mathcal{F}\left(x / l_{\perp}, L / l_{\perp}\right),
$$

where $a_{0}$ is the vortex spacing and $\rho_{0}=\left(n_{0} \phi_{0} / c\right)^{2}\left(1 / \gamma_{0}\right)$ is the Bardeen-Stephen resistivity of noninteracting flux 
lines, with $\gamma_{0}$ a bare friction. A scaling form for the resistivity $\rho(T, L)=\Delta V /(L J)$, with $\Delta V$ the net voltage drop across the channel, is easily obtained by integrating Eq. (3), with the result,

$$
\rho(T, L)=\rho_{f}(T) f\left(L / l_{\perp}\right)
$$

with $f(x)=\frac{1}{x} \int_{0}^{x} d u \mathcal{F}(u, x)$ a scaling function and $\rho_{f}(T)$ the bulk resistivity,

$$
\rho_{f}(T)=\rho_{0}\left(\frac{l_{\perp}}{a_{0}}\right)^{2-z} \equiv\left(\frac{n_{0} \phi_{0}}{c}\right)^{2} \frac{1}{\gamma} .
$$

In the second line of Eq. (5) the dependence on the Bose glass correlation length $l_{\perp}$ has been incorporated in a renormalized friction coefficient $\gamma=\gamma_{0}\left(\frac{l_{\perp}}{a_{0}}\right)^{z-2}$ that di-

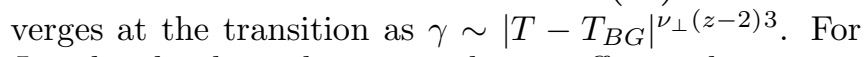
$L \gg l_{\perp}$, the channel geometry has no effect and one must recover the bulk result, leading to $f(x \gg 1) \sim 1$.

The scaling function $\mathcal{F}$ can be determined by assuming that the long wavelength electric field of Eq. (3) is described by hydrodynamic equations 15 . For simple geometries where the current is applied in the $a b$ plane and the flow is spatially homogeneous in the $z$ direction, these reduce to a single equation for the coarse-grained flux liquid flow velocity $\mathbf{v}(\mathbf{r}) 17$

$$
-\gamma \mathbf{v}+\eta \nabla_{\perp}^{2} \mathbf{v}+\mathbf{f}_{L}=0,
$$

The second term in Eq. (5) is the flux liquid viscosity $\eta(T, H)$ and represents the viscous drag arising from intervortex interactions and entanglement. Finally, $\mathbf{f}_{L}=-\frac{1}{c} n_{0} \phi_{0} \hat{\mathbf{z}} \times \mathbf{J}$ is the Lorentz force density driving the flux motion. Intervortex interaction at the Bose-glass boundaries translates into a no-slip boundary condition for the flux liquid flow velocity. By preventing the free flow of flux liquid, the Bose glass boundaries can significantly decrease the macroscopic flux-flow resistivity of the superconductor. Once the velocity field is obtained by solving Eq. (6) with suitable boundary conditions, the electric field profile in the superconductor is found immediately from $\mathbf{E}(\mathbf{r})=\frac{n_{0} \phi_{0}}{c} \hat{\mathbf{z}} \times \mathbf{v}(\mathbf{r})$. It is instructive to rewrite Eq. (6) as an equation for the local electric field,

$$
-\delta^{2} \nabla_{\perp}^{2} \mathbf{E}+\mathbf{E}=\rho_{f} \mathbf{J},
$$

where $\delta=\sqrt{\eta / \gamma}$ is the viscous length. When the first term on the right hand side is absent, i.e., the flux liquid viscosity is small, this equation of "viscous electricity" reduces to Ohm's law with flux flow resistivity given by the bulk value, $\rho_{f}(T)$. Interactions, however, make the viscous drag important and as a result the electrodynamics of flux-line liquids is highly nonlocal near the Bose glass transition.

The solution of the hydrodynamic equation for the simple channel geometry sketched in Fig. 1, with a homogeneous current $\mathbf{J}=-\hat{\mathbf{x}} J$ applied across the channel, is given by

$$
E(x, L)=\rho_{f} J\left[1-\frac{\cosh (x / \delta)}{\cosh (L / 2 \delta)}\right],
$$

and is shown in Fig. 1. Upon comparing Eq. (8) to Eq. (3), we see that the quantity in square brackets in Eq. (8) is the scaling function $\mathcal{F}$ and find that the viscous length $\delta$ is in fact the Bose glass length $l_{\perp}$. As the friction diverges at $T_{B G}$ according to $\gamma \sim\left|T-T_{B G}\right|^{-\nu_{\perp}(z-2)}$, this identification immediately gives that the flux liquid shear viscosity also diverges at the Bose glass transition with

$$
\eta=l_{\perp}^{2} \gamma \sim\left|T-T_{B G}\right|^{-\nu_{\perp} z} .
$$

The scaling form for the resistivity is obtained by integrating Eq. (8), with the result

$$
\rho(T, L)=\rho_{f}(T)\left[1-\frac{2 l_{\perp}}{L} \tanh \left(\frac{L}{2 l_{\perp}}\right)\right] .
$$

If $l_{\perp} \ll L$, we recover the bulk result of Eq. (5), $\rho(T, L)=\rho_{f}(T) \sim\left|T-T_{B G}\right|^{\nu_{\perp}(z-2)}$. Near the transition, where $l_{\perp} \gg L$, the resistivity depends on the channel width and is controlled by the shear viscosity, with

$$
\rho(T, L) \simeq \frac{\rho_{f} L^{2}}{12 l_{\perp}^{2}}=\left(\frac{n_{0} \phi_{0}}{c}\right)^{2} \frac{L^{2}}{12 \eta(T)} \sim L^{2}\left|T-T_{B G}\right|^{\nu_{\perp} z} .
$$

This strong divergence of the viscosity is precisely the kind of behavior expected at a liquid-glass transition. In this sense the Bose glass transition is an example of a glass transition that is well understood theoretically and where precise predictions are available.

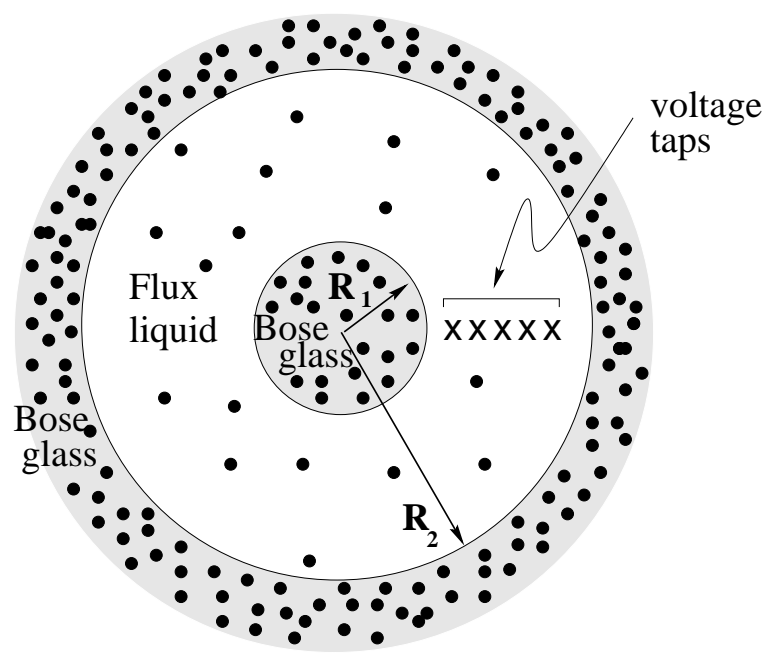

FIG. 3. Top view of the Corbino disk geometry with Bose glass contacts. The magnetic field is out of the page. The vortex array is in the Bose glass state in the inner and outer densely dotted regions and in the flux liquid state in the weakly irradiated annular region. A radial driving current drives flux motion in the azimuthal direction, and the voltage taps allow the electric field profile to be determined. 
Another important patterned geometry is the Corbino disk, recently used by López et al. for defect-free materials 16 to reduce boundary effects in the flux flow measurements. Here we propose fabrication of a Corbino disk with Bose glass inner and outer contacts sketched in Fig. 3. A current $I$ injected at the outer boundary and extracted at the inner boundary yields a radial current density $\mathbf{J}(r)=-\frac{I}{2 \pi\left(R_{2}-R_{1}\right)} \frac{\hat{\mathbf{r}}}{r}$ that drives vortex motion in the azimuthal direction. The electric field induced by flux motion is radial, $\mathbf{E}(\mathbf{r})=-E(r) \hat{\mathbf{r}}$, and its magnitude is obtained by solving Eq. (7) in a cylindrical geometry, with the result,

$$
E(r)=\frac{\rho_{f} I}{2 \pi\left(R_{2}-R_{1}\right) l_{\perp}}\left[\frac{l_{\perp}}{r}+c_{1} I_{1}\left(\frac{r}{l_{\perp}}\right)+c_{2} K_{1}\left(\frac{r}{l_{\perp}}\right)\right],
$$

where

$$
\begin{aligned}
& c_{1}=\frac{K_{1}\left(\rho_{2}\right) / \rho_{1}-K_{1}\left(\rho_{1}\right) / \rho_{2}}{K_{1}\left(\rho_{1}\right) I_{1}\left(\rho_{2}\right)-K_{1}\left(\rho_{2}\right) I_{1}\left(\rho_{1}\right)} \\
& c_{2}=\frac{I_{1}\left(\rho_{1}\right) / \rho_{2}-I_{1}\left(\rho_{2}\right) / \rho_{1}}{K_{1}\left(\rho_{1}\right) I_{1}\left(\rho_{2}\right)-K_{1}\left(\rho_{2}\right) I_{1}\left(\rho_{1}\right)},
\end{aligned}
$$

with $\rho_{1,2}=R_{1,2} / l_{\perp}$ and $I_{1}(x)$ and $K_{1}(x)$ Bessel functions. The electric field profiles are shown in Fig. 4. The resistivity is defined in terms of the net voltage drop $\Delta V_{12}$ between the inner and outer radii as $\rho\left(T, R_{1}, R_{2}\right)=$ $\Delta V_{12} /\left[I / 2 \pi\left(R_{2}-R_{1}\right)\right]$. Near the Bose glass transition, where $l_{\perp} \gg R_{2}, R_{1}$, we find

$$
\rho \simeq \frac{\left(n_{0} \phi_{0} / c\right)^{2}}{4 \eta(T)}\left\{\frac{R_{2}^{2}-R_{1}^{2}}{2}-\frac{4 R_{1}^{2} R_{2}^{2}\left[\ln \left(R_{2} / R_{1}\right)\right]^{2}}{R_{2}^{2}-R_{1}^{2}}\right\} .
$$

As in the channel geometry, the resistivity at the transition is completely determined by the diverging viscosity and the geometrical parameters of the channel.

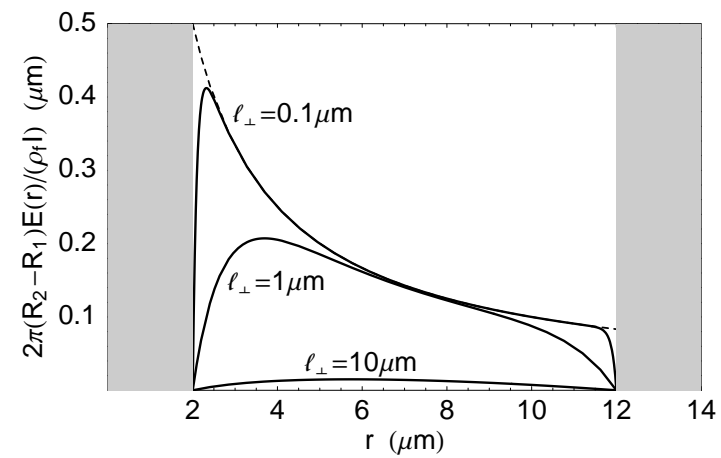

FIG. 4. The electric field profile for the Corbino disk geometry (Eq. (12)), with $R_{1}=2 \mu \mathrm{m}$ and $R_{2}=12 \mu \mathrm{m}$, with a width $R_{2}-R_{1}=10 \mu \mathrm{m}$. The vertical axis represents the reduced field $2 \pi E(r) /\left(\rho_{f} I\right)$ in $\mu m$. The dashed line is the electric field for vanishing viscosity, $E_{0}(r)=\frac{\rho_{f} I}{2 \pi\left(R_{2}-R_{1}\right)} \frac{1}{r}$. The shaded regions represent the Bose glass contacts.
Experiments with patterned geometries near the Bose glass transformation provide an exciting opportunity to probe viscous behavior near a second order glass transition. A similar scaling analysis leads to predictions for the additional viscosities which characterize the dynamics of vortex matter 151 18. For example, the viscous generalization of Ohm's law for transport parallel to the applied field reads

$$
-\delta_{\|}^{2} \partial_{z}^{2} E_{\|}-\delta_{\perp}^{2} \nabla_{\perp}^{2} E_{\|}+E_{\|}=\rho_{\|} J_{\|},
$$

with $\rho_{\|} \sim l_{\perp}^{-z}, \delta_{\|} \sim l_{\|}$, and $\delta_{\perp} \sim l_{\perp}$.

This work was supported by the National Science Foundation at Syracuse through Grants No. DMR9730678 and DMR98-05818 and at Harvard through Grant No. DMR97-14725, and by the Harvard Materials Research Science and Engineering Center through Grant No. DMR98-09363.

${ }^{1}$ Crabtree, G.W., and Nelson, D.R., Physics Today 50, No. 4, 38-45 (1997), and references therein.

${ }^{2}$ Fisher, M.P.A., Vortex-glass superconductivity: A possible new phase in bulk high- $T_{c}$ oxides, Phys. Rev. Lett. 62, 1415-1418 (1989); Fisher, D.S., Fisher, M.P.A., and Huse, D.A., Thermal fluctuations, quenched disorder, phase transitions, and transport in type-II superconductors, Phys. Rev. B 43, 130-159 (1991).

${ }^{3}$ Nelson, D.R., and Vinokur, V.M., Boson localization and pinning by correlated disorder in high-temperature superconductors, Phys. Rev. Lett. 68, 2398-13401 (1992); Boson localization and correlated pinning of superconducting vortex arrays, Phys. Rev. B 48, 13 060-13 097 (1993).

${ }^{4}$ Budhani, R.C., Suenaga, M., and Liou, H.S., Giant suppression of flux-flow resistivity in heavy-ion irradiated $\mathrm{Tl}_{2} \mathrm{Ba}_{2} \mathrm{Ca}_{2} \mathrm{Cu}_{3} \mathrm{O}_{10}$ films: Influence of linear defects on vortex transport, Phys. Rev. Lett. 69, 3816-3819 (1992).

${ }^{5}$ Konczykowski, M., et al., Effect of $5.3-\mathrm{GeV} \mathrm{Pb-ion} \mathrm{irradi-}$ ation on irreversible magnetization in Y-Ba-Cu-O crystals, Phys. Rev. B 44, 7167-7170 (1991).

${ }^{6}$ Civale, L., et al., Vortex confinement by columnar defects in $\mathrm{YBa}_{2} \mathrm{Cu}_{3} \mathrm{O}_{7}$ crystals: Enhanced pinning at high fields and temperatures, Phys. Rev. Lett. 67, 648-651 (1991).

${ }^{7}$ See, e.g., Grigera, S.A., et al., Bose-Glass Phase in Twinned $\mathrm{YBa}_{2} \mathrm{Cu}_{3} \mathrm{O}_{7-\delta}$, Phys. Rev. Lett. 81, 2348-2351 (1998).

${ }^{8}$ Nelson, D.R., Vortex entanglement in high- $T_{c}$ superconductors, Phys. Rev. Lett. 60, 1973-1976 (1988).

${ }^{9}$ For simplicity, we confine our attention here to fields less that the "matching field" $B_{\phi}=n_{\text {pin }} \phi_{0}$, with $n_{\text {pin }}$ the areal density of columnar pins and $\phi_{0}=\hbar c / 2 e$ the flux quantum.

${ }^{10}$ Nelson, D.R., and Radzihovsky, L., Longitudinal current dissipation in Bose-glass superconductors, Phys. Rev. B 54, R6845-R6848 (1996). 
${ }^{11}$ Fisher, M.P.A., Weichman, P.B., Grinstein, G., and Fisher, D.S., Boson localization and the superfluid-insulator transition, Phys. Rev. B 40, 546-570 (1989).

${ }^{12}$ Wallin, M., and Girvin, S.M., $I-V$ characteristics of hightemperature superconductors with columnar defects, Phys. Rev. B 47, 14 642-14 645 (1993); Lidmar, J., and Wallin, M., Critical properties of Bose-glass superconductors, private communication; Lidmar, J., Doctoral Dissertation, Royal Institute of Technology, University of Stockholm, Sweden.

${ }^{13}$ A diverging shear viscosity at the vortex freezing transition predicted by the theory of two-dimensional melting has been observed in superconducting films by Theunissen, M.H., Van der Drift, E., and Kes, P.H., Size Effects in Flow of Flux-Line Solids and Liquids, Phys. Rev. Lett. 77, 159-162 (1996).

${ }^{14}$ Pastoriza, H., and Kes, P.H., Direct shear probe of vortex lattice melting in $\mathrm{Bi}_{2} \mathrm{Sr}_{2} \mathrm{CaCu}_{2} \mathrm{O}_{8}$ single crystals, Phys.
Rev. Lett. 75, 3525-3528 (1995).

${ }^{15}$ Marchetti, M.C., and Nelson, D.R., Hydrodynamics of flux liquids, Phys. Rev. B 42, 9938 (1990); Dynamics of flux-line liquids in high- $T_{c}$ superconductors, Physica C 174, 40-62 (1991).

${ }^{16}$ López, D., et al., Spatially resolved dynamic correlation in the vortex state of high temperature superconductors, submitted to Nature; Mazilu, A., et al., Experimental comparison of the effect that bulk pinning and surface barriers have on vortex motion in the vortex liquid state of $\mathrm{Bi}_{2} \mathrm{Sr}_{2} \mathrm{CaCu}_{2} \mathrm{O}_{8}$ single crystals, Phys. Rev. B 58, R8913R8916 (1998).

${ }^{17}$ For simplicity we have neglected here the small component of the frictional force on the flux liquid normal to the flow velocity, which is responsible for the Hall effect.

18 Mou, C.-Y., Wortis, R., Dorsey, A.T., and Huse, D.A., Nonlocal conductivity in type-II superconductors, Phys. Rev. B 51, 6575-6587 (1995). 